\title{
Some Peculiarities of Photo-structural Transformations in Amorphous Chalcogenide Glassy Semiconductor Films
}

\author{
O. Prikhodko, N. Almasov, and N. Korobova, Member, KIMICS
}

\begin{abstract}
The absence of deep traps for electrons in the spectrum of $\mathrm{As}_{40} \mathrm{Se}_{30} \mathrm{~S}_{30}$ localized states films obtained by ion sputtering was determined. Bipolar drift of charge carriers was found in amorphous $\mathrm{As}_{40} \mathrm{Se}_{30} \mathrm{~S}_{30}$ films of chalcogenide glassy semiconductors, obtained by ion-plasma sputtering of high-frequency, unlike the films of these materials obtained by thermal evaporation.
\end{abstract}

Index Terms - Bipolar drift, charge carriers, amorphous films, chalcogenide glassy semiconductors, ion-plasma sputtering of high-frequency, thermal evaporation.

\section{INTRODUCTION}

MAJOR carriers in the semiconductor in the electric field absence usually commit random thermal motion changing its direction as a result of collisions with impurity ions such as donors or acceptors. Collisions are not to be taken literally, such as the collision of two billiard balls. We discuss about the interaction of electric fields charge of mobile carriers and fixed impurity ions. Although atoms of the basic substance are more numerous in these processes they almost do not participate because of their electrical neutrality. The higher the concentration of impurities in semiconductors, the shorter free path of carriers value [1].

Ordered component like a drift is superimposed in the electric field on the thermal motion of carriers. Continuing the chaotic motion, the carriers begin to move along the lines of the electric field in the direction determined by the sign of the carrier. Carrier mobility relates velocity and field strength. In other words, the value of mobility - is the rate of carriers in the unit field [2]. The linear dependence is preserved only at relatively low values of the field. In strong fields, saturation of the drift velocity was noted. This is also an important characteristic of semiconductors in terms of their use at high frequencies. Note also that with increasing doping concentration of scattering centers increases and mobility decreases. Another kind of ordered motion - diffusion takes place along with the drift.

Manuscript received July 22, 2011; revised August 5, 2011; accepted August 18, 2011.

Natalya Korobova is with the Physics Department of Kazakh National University, al-Farabi 71, Almaty, 050040, Kazakhstan (Email: Nataliya.Korobova@kaznu.kz).
Clash of the carrier motion in the direction of higher concentration are more likely than when the carrier moving toward low concentrations. Because of this, carriers will be distributed from the region of high concentration to a region with low concentration. The drift mobility of charge carriers in non-crystalline semiconductors usually is determined from the study of the transient photocurrent of no equilibrium charge carriers. Development of this technique belongs to Spiru $[1,3]$. The technique allows to separately determining the drift mobility of electrons $\mu_{\mathrm{n}}$ and holes $\mu_{\mathrm{p}}$ in materials in which transit time of carriers is less than the dielectric relaxation.

\section{EXPERIMENTAL RESULTS}

Sample of "sandwich", where analyzed material layer is placed between two electrodes was used in these experiments. One of the electrodes is translucent in the case of optical excitation of charge carriers. For the test sample is applied either constant voltage or voltage pulse. Near the upper semitransparent electrode in the contact region with a short pulse of light creates a strongly absorbing high concentrations of charge carriers. Electrons or holes drift across the sample depending on the polarity of the applied voltage. Technique for measuring the drift mobility of charge carriers in materials with a characteristic high resistance (usually $\rho>10^{7} \mathrm{Ohm}$ $\mathrm{cm})$ used most effectively. Injected carriers are not screened at the time of charge carriers flight $\left(t_{f 1}\right)$ smaller than the dielectric relaxation time $\left(\tau_{\mathrm{M}}=\varepsilon \varepsilon_{0} / \sigma\right)$. Therefore it is possible to separate determination of the electron and hole mobility. In the case of material with a high concentration of intrinsic carriers, excess carriers are screened and, therefore, appear only the minority carriers. Typically, the experiments on the drift mobility are conducted under conditions of weak injection, which corresponds to the condition:

$$
e N_{\mathrm{o}}<<C V
$$

where: e - electron charge, $\mathrm{N}_{\mathrm{o}}$ - injected carriers concentration, $\mathrm{C}$ - sample capacitance.

In this case, the external current corresponds to a change of charge in the sample over time:

$$
I=d Q / d t=e N_{0} \mu U / l^{2} .
$$


The corresponding voltage drop across the load resistance (Figure 1 and $2 \mathrm{a}$ ) is:

$$
\Delta U=I R=e N_{\mathrm{o}} R \mu U / l^{2},
$$

where $\mu$. carrier drift mobility, U - voltage across the sample, 1 - thickness of the sample.

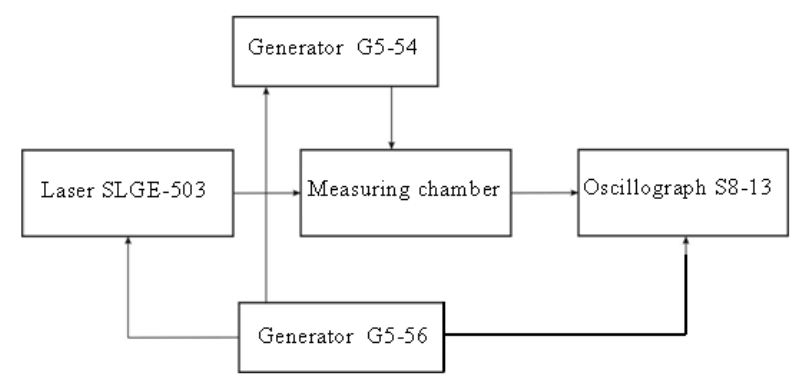

Fig. 1. Schematic apparatus diagram for measuring the carrier drift mobility

The actual shape of the photocurrent pulses corresponding to the charge carriers drift is a gradual decrease in current over time, which does not allow direct determination of flight time (Figure 2b).

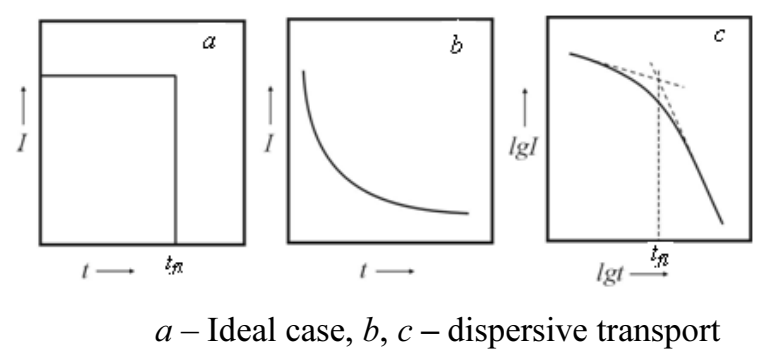

Fig. 2. Transient photocurrent carriers in non-crystalline semiconductors.

In this case, transit time determination of structure less photocurrent decay curves was transformed into a double logarithmic scale $\operatorname{lgI} \sim$ lgt. Flight time was obtained using moment time of temporary changing nature of photocurrent decay (Figure 2 a) [3]. Such defined $t_{f 1}$ value corresponds to the time out of the sample's drifting pack carriers of center of gravity.

Modified depending decline of the transient photocurrent versus time was approximated by the power functions [4]:

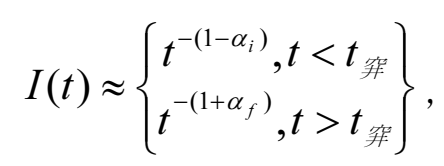

where $\alpha_{\mathrm{i}}=\alpha_{\mathrm{f}}=\alpha$-dispersion parameters characterizing the dispersion degree of carrier transport and, consequently, the pulse shape of the transient photocurrent. With a known samples thickness (d) and electric field (E), the drift mobility of charge carrier $\mu$ is determined as follows:

$$
\mu_{d}=\mu_{0} \frac{d^{2}}{t_{\text {突 }} \cdot A} .
$$

Form deviation of the pulse transient photocurrents from the ideal may be due to the peculiarities of carrier transport, and also the fact that the sample is moved not sufficiently narrow package carrier. The presence in the material of deep traps, the release time of which is greater than the transit time, reduces the concentration of charge carriers during their movement in the sample. Carrier concentration changing, as a rule, described by exponential function of the form:

$$
N=N_{\mathrm{o}} \exp (t / \tau)
$$

where - $\tau$ the effective life time of nonequilibrium carriers, which characterizes the time during which carriers are involved in charge transfer to the capture by the traps. To obtain the transient photocurrent undistorted pulse shape, so in this case the necessary condition will be $t_{\mathrm{fl}}<\tau$. To do this, necessary make measure in a sufficiently strong electric fields $\left(\mathrm{E} \sim 10^{5} \mathrm{~V} / \mathrm{cm}\right)$. Another most important factor in these experiments is the formation of a sufficiently small package carrier. Expansion (dispersion) of the carriers package may be due to various reasons, such as carrier diffusion, or using weakly absorbed radiation $\left(\alpha^{-1} \geq d\right)$ and a large pulse of exciting $\operatorname{radiation}\left(t_{\text {pulse }}\right)$. Therefore, for correct charge carriers drift mobility measurement and characteristics of the transfer determination is necessary to ensure the following conditions [5]:

$$
\alpha^{-1} \geq d, \quad t_{\text {имп }}<<t_{\text {пр }},
$$

where $\alpha$-light absorption coefficient.

In addition, the significant effect on the blur of drifting carrier packet, and consequently on the shape of the transient photocurrents pulse provides electrode material and, above all, the electrode material through which photo-injection carriers in the sample was done. This is due to the formation of negative space charge in the electrode region, which causes uneven distribution of electric field on the model [5]. For the space charge formation in the electrode region it is necessary some time $t_{\mathrm{f}}$. Consequently, using the pulsed voltage and short time delay between the filing of the voltage pulse on the sample and the time photo-injection carriers can substantially weaken the space charge influence on the measurement process. For this reason the synchronizing, providing the amount of time delay in the interval $t_{\mathrm{d}}$ : 


$$
R C<t_{\mathrm{d}}<t_{\mathrm{f}}
$$

where $R C$-time constant of the measuring circuit.

In our experiments, the RC is less than 0.2 microseconds, and the experimentally chosen delay time was $100 \mu \mathrm{s}$. Signal corresponding to the carriers drift through the sample, with the load resistance applied to the input storage oscilloscope C8-13 with maximum sensitivity $5 \cdot 10^{-4} \mathrm{~V} /$ div. Pulse processing transient photocurrents have been slow-scan signal for the entire duration. Further processing of experimental data was carried out on the PC. Measurements were performed in single pulse excitation. Sample holder was placed in a darkened and carefully screened special chamber, the sample temperature monitored by a copper-constantan thermocouple. Al was used as an electrode material for "sandwich" structure. Films thickness was in the ranged from 0.5 to 10 microns.

\section{RESULTS AND DISCUSSIONS}

Study results of the charge carriers drift mobility in the $\mathrm{As}_{40} \mathrm{Se}_{30} \mathrm{~S}_{30}$ RF-films identified a number of significant differences in the transport processes in these films from the bulk samples and TE films of this composition. In the $\mathrm{As}_{40} \mathrm{Se}_{30} \mathrm{~S}_{30}$ RF-films mobile carriers are the carriers of both signs. Electrons and holes drift mobility magnitude were approximately the same $\mu_{\mathrm{p}} \approx \mu_{\mathrm{n}} \approx 10^{-5} \mathrm{~cm}^{2} /(\mathrm{V} \cdot \mathrm{s})$ at $\mathrm{T}=300 \mathrm{~K}$ and $\mathrm{E}=10^{5} \mathrm{~V} / \mathrm{cm}$. Dependence of $\mu_{\mathrm{p}} \approx \mu_{\mathrm{n}}$ versus the electric field can be approximated by an exponential function $\mu_{\mathrm{p}}, \mu_{\mathrm{n}} \sim \exp \left(\mathrm{E} / \mathrm{E}_{\mathrm{o}}\right)$, where $\mathrm{E}_{\mathrm{o}}=10^{5}$ $\mathrm{V} / \mathrm{cm}$. In the studied temperature range the electrons and holes drift mobility increases with temperature exponentially with equal values of activation energies $\varepsilon_{\text {мр }}$ $=\varepsilon_{\mu \mathrm{n}}=(0,53 \div 0,57) \mathrm{eV}$. These experimental facts suggest that $\mathrm{As}_{40} \mathrm{Se}_{30} \mathrm{~S}_{30} \mathrm{RF}$ films have bipolar drift of charge carriers. Experimentally observed field dependence on the conductivity and drift mobility can be interpreted in a model of charged defects $[1,5]$. In the model of charged defects, the charge carriers drift mobility is limited by trapping at charged centers $\mathrm{D}^{+}$and $\mathrm{D}^{-}$. For example, if the hole drift mobility will be limited by trapping at the state of $\mathrm{D}^{-}$, then for the field dependence of hole drift mobility can be expected depending on such Poole-Frenkel law. Activation of charge carriers in the dark conductivity by two successive reactions: $\mathrm{D}^{+}+\mathrm{D}^{-} \rightarrow 2 \mathrm{D}^{\circ}$ and $\mathrm{D}^{\circ} \rightarrow \mathrm{p}+\mathrm{D}^{+}$ and $\mathrm{D}^{\circ} \rightarrow \mathrm{e}+\mathrm{D}^{-}$. If we assume that the first process does not depend on the field, the latter two processes and determine the drift mobility. Then, the equilibrium concentration of charge carriers will depend on the same factors as the drift mobility.

In our paper the model close to gauss distribution was proposed for identification the energy distribution of localized states in amorphous $\mathrm{As}_{40} \mathrm{Se}_{30} \mathrm{~S}_{30}$ films:

$$
n(\varepsilon)=2 N_{t}\left[\left(\varepsilon-\varepsilon_{t}\right) / \varepsilon_{o}^{2}\right] \exp \left[-\left(\varepsilon-\varepsilon_{t}\right) / \varepsilon_{o}^{2}\right]
$$

where $-\varepsilon_{t}$ energy corresponding to the distribution maximum, $\varepsilon_{o}$ - energy that characterizes the distribution width.

As shown in [5], the function of this form of distribution gives the following expressions for the dispersion parameters:

$$
\begin{gathered}
\alpha_{i}=2\left(k T / \varepsilon_{o}\right)\left[\ln \left(d / \sqrt{2} \mu_{o} \tau_{o} E\right)\right]^{1 / 2}\left\{1+\left[1 / 2 \ln \left(d / \sqrt{2} \mu_{o} \tau_{o} E\right)\right]\right\} \\
\alpha_{f}=2\left(k T / \varepsilon_{o}\right)\left[\ln \left(d / \sqrt{2} \mu_{o} \tau_{o} E\right)\right]^{1 / 2}\left\{1-\left[1 / 2 \ln \left(d / \sqrt{2} \mu_{o} \tau_{o} E\right)\right]\right\} \\
\alpha_{T}=2\left(k T / \varepsilon_{o}\right)\left[\ln \left(d / \sqrt{2} \mu_{o} \tau_{o} E\right)\right]^{1 / 2}
\end{gathered}
$$

And for the activation energy:

$$
\varepsilon_{\mu}=\varepsilon_{t}+\varepsilon_{o}\left[\ln \left(d / \sqrt{2} \mu_{o} \tau_{o} E\right)\right]^{1 / 2}
$$

where $\mu_{o}$ - mobility and $\tau_{o}$ lifetime of free carriers, respectively.

Comparison of the calculated and experimental data showed that good correspondence between them and achieved at values $\varepsilon_{\mathrm{o}}=0.16 \mathrm{eV}$ and $\mu_{\mathrm{o}} \tau_{\mathrm{o}} \approx 10^{-10} \mathrm{~cm}^{2} / \mathrm{V}$. Activation energies values substitution for different film thicknesses and electric fields, allowed us to determine the position of the distribution function $\varepsilon_{\mathrm{t} 1}=\varepsilon_{\mathrm{t} 2} \approx 0,52$ $\mathrm{eV}$. Maxima of the localized states density distribution for both electrons and holes in the $\mathrm{As}_{40} \mathrm{Se}_{30} \mathrm{~S}_{30}$ RF-films shifted deep into the gap band on $\varepsilon_{\mathrm{t}} \approx 0,52 \mathrm{eV}$. Thus, the results of localized states spectrum identification in $\mathrm{As}_{40} \mathrm{Se}_{30} \mathrm{~S}_{30}$ films has been shown that the experimental data can be explained by the assumption about the quasidiscrete levels broadening of localized states with $\varepsilon_{\mathrm{t}} \approx$ $0,52 \mathrm{eV}$. It is approximated by a Gaussian density of states. Distribution peculiarities in this case consist of in the fact that they are located symmetrically about mid gap (Fig. 3).

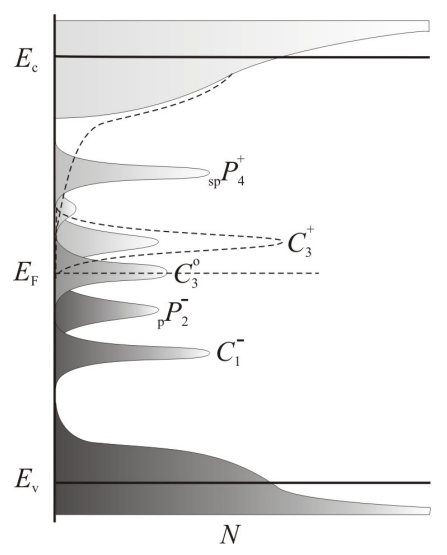

Fig. 3. Energy distribution model of the localized states in the $\mathrm{As}_{40} \mathrm{Se}_{30} \mathrm{~S}_{30}$ RF-films 
Thus, the basic laws of electronic properties change for chalcogenide glassy semiconductors are intrinsically linked to structure differences using different method of preparation. The extent and nature of the observed changes in the films structure and properties of the same composition depend on the formed defect types and concentrations. It is apparently determined by the molecular structure of the CGS deposited layers. In the usual thermal evaporation method, for example, $\mathrm{As}_{40} \mathrm{Se}_{30} \mathrm{~S}_{30}$ condensate film having a structure approaching the structure of the monolithic material [1], which causes almost complete identity of their electronic properties.

\section{CONCLUSION}

CGS films obtained by thermal evaporation in vacuum (TE-film) and ion-plasma high-frequency sputtering (RFfilms) were investigated.

It was founded, that electronic properties of noncrystalline semiconductors based on films of arsenic chalcogenide glassy semiconductors, obtained by ionplasma RF sputtering and thermal evaporation in a vacuum, greatly depend on how they were prepared.

In amorphous $\mathrm{As}_{40} \mathrm{Se}_{30} \mathrm{~S}_{30}$ films, obtained by ion plasma RF sputtering, in contrast to the films of these materials obtained by thermal evaporation, observed bipolar photoconductivity and bipolar drift of charge carriers. This is due to the lack of deep traps for electrons in the spectrum of localized states of the films obtained by ion sputtering.

External influences on the films such as light exposure and annealing were accompanied by changes in the optical band gap and the atomic structure extent of a medium-range order. In the cycles of "light exposure annealing", these changes are reversible in nature, i.e. such fundamental parameters as the band gap of the studied non-crystalline semiconductor films has been determined not only neighbors, but medium-range order of atomic structure.

The electron and hole transport processes in $\mathrm{As}_{40} \mathrm{Se} 30 \mathrm{~S}_{30}$ films of ternary stoichiometric composition, obtained by ion-plasma sputtering of high-frequency, were controlled by trapping at discrete levels of intrinsic structural defects that were located in energy at the same depth from the edge of the allowed energy bands. Levels energy blurring can be approximated by a Gaussian distribution law, with peaks at $\varepsilon_{t 1}=\varepsilon_{t 2}=0,52 \mathrm{eV}$ and the distribution width equal to $0.16 \mathrm{eV}$.

Obtained results are very important for the development of the fundamental concepts of electronic processes in non-crystalline semiconductors and open up new possibilities for obtaining films of these materials with desired electronic properties. It leads to almost complete identity of the main characteristics of the electrons and holes transfer in the $\mathrm{As}_{40} \mathrm{Se}_{30} \mathrm{~S}_{30} \mathrm{RF}$-films.

\section{ACKNOWLEDGMENT}

This work was supported by Ministry of Education and Science of the Republic of Kazakhstan Fundamental Investigation grant (FI-13.10) and was done in the Scientific Investigation Institute of Experimental and Theoretical Physics.

\section{REFERENCES}

[1] Mott and E.A. Davis. Electronic Processes in Non-Crystalline substance. Moscow: Mir, 1982, 560p.

[2] Amorphous Semiconductors / Ed. Brodsky M. - Moscow: Mir, 1982, $-320 \mathrm{p}$.

[3] Electronic phenomena in chalcogenide glassy semiconductors /Ed. Tsendin K.D. Moscow: Science 1996, 486p.

[4] Scher H., Montroll E. W. Anomalous transit-time dispersion in amorphous solids // Phys. Rev. - 1975. - Vol.12, № 6. - P. 2455 2477.

[5] E., Kozakova L. Drift charge carriers in chalcogenide glasses/ in Electronic phenomena in chalcogenide glassy semiconductors.- Ed. Tsendin K.D. Moscow: Science 1996, 486p.

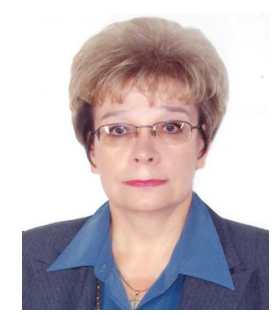

Korobova Natalya - Almaty, October 1953 Professor of Kazak National University named al-Farabi. Ph.D. in Technical Science (1988, St.-Petersburg, Russia), Dr.Sc. on Chemistry (1999, Almaty, Kazakhstan).Major fields of study - material science, sol-gel technology, SHS technology, new materials for electronics. 